\title{
Mathematical Modeling of the Dynamics of
}

\section{a Shovel with Flexible Coupling}

\author{
Dario Friso \\ Department of Land, Environment, Agriculture and Forestry - TESAF \\ Research Unit: Agricultural and Food Engineering \\ University of Padova, Viale dell'Università 16, Legnaro 35020, Padova, Italy
}

\section{Hans-Peter Haller}

Ratschings 39040, Bozen, Italy

Martin Zingerle

Zingerlemetal AG

Natz/Schabs 39040, Bozen, Italy

\begin{abstract}
Copyright (C) 2015 Dario Friso, Hans-Peter Haller and Martin Zingerle. This article is distributed under the Creative Commons Attribution License, which permits unrestricted use, distribution, and reproduction in any medium, provided the original work is properly cited.
\end{abstract}

\begin{abstract}
To study the possible ergonomic and energetic advantages on the use of a shovel with a flexible coupling, a dynamic analysis of the motion of the blade together with the lifted raw material was conducted. By this dynamic analysis, ordinary differential equations were set up. Therefore the closed-form solutions were found to obtain useful equations for simulation. The application of mathematical modeling both to the blade with elastic coupling and to the traditional one, allowed to quantify the zeroing of the operator effort in the second half of the blade lifting. However, in the first part of the lifting an effort increase occurs, but in this first phase the operator can take advantage from the support on the thigh, thus lightening the load on the spine.
\end{abstract}

Keywords: Shovel; Mathematical modeling; ODE; Energy and work; Dynamic analysis; Ergonomics; Agricultural and forest engineering 


\section{Introduction}

Shovel are still widely used for manual work of digging and material transfer in agriculture, forest and construction industry [11]. It is a hard work with high energy consumption and high musculoskeletal stress, particularly for the spine.

In a recent research [2] laboratory tests were carried out to simulate the conditions of work with the shovel. The musculoskeletal loads for the lifting work of 5 different types of material (soil, sand, gravel, pebbles and mortar) were measured. First, however, masses shoveling, execution times and motion kinematics of workers were quantified. The mean value of lifted mass was $7.68 \pm 1.65 \mathrm{~kg}$. The mean value of entire operation time was $2.99 \pm 1.07 \mathrm{~s}$.

The kinematic analysis of worker movements allows to identify the average elevation time of mass by shovel equal to $0,8 \pm 0.16 \mathrm{~s}$. Finally, the mean compression force on the inter-vertebral disc was $3,5 \div 6,25 \mathrm{kN}$.

Considering these information, a shovel with a flexible joint in the handle was built and patented [3]. The flexible coupling have to flex in vertical plane but not in horizontal one (fig. 1).

The aim of this paper is to study the motion dynamics of material mass lifted by the worker with the shovel. For a comparison, the dynamic analysis was conducted both for a shovel without spring and for one with the spring at two different stiffness.

The dynamic analysis will be carried out by integration of ODE looking for closed solutions, as made in previous researches [4, 8, 12 and 13], having the same objective of obtaining equations to be easily implemented both in a control system and in spreadsheet for simulation. Finally the dynamic analysis will be also conducted with the aim to describe the energetic aspect.

\section{Dynamic analysis}

\subsection{Shovel without flexible coupling}

The blade of figure 1 is equipped with a traditional handle (without flexible coupling). The operator moves the blade through the handle. Therefore the blade constitutes a mobile support for the mass $m$. It is assumed that the blade runs a vertical trajectory of the coordinate $z$ with reference to ground (inertial frame) as shown in figure 1 (right).

It is a sinusoidal trajectory vs. time, represented by the following equation:

$$
z=\frac{z_{\max }}{2}-\frac{z_{\max }}{2} \cos \omega t
$$



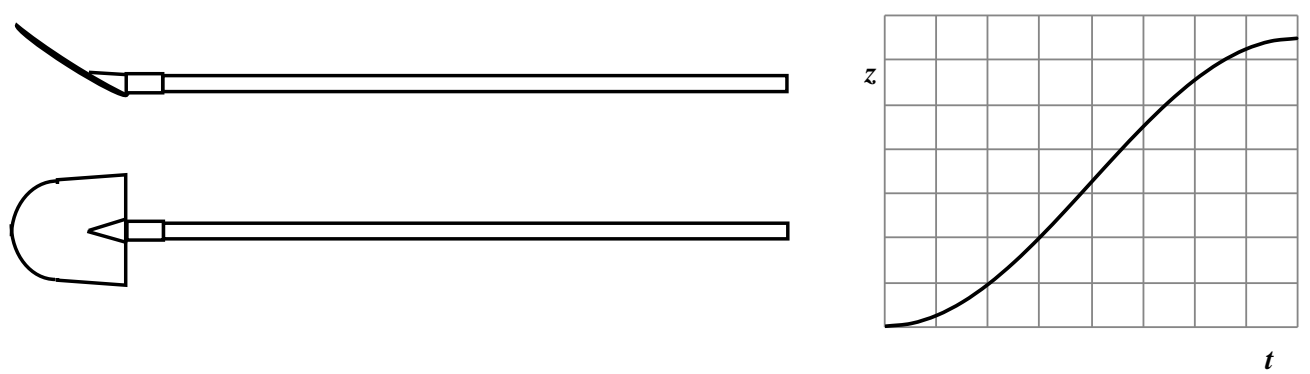

Fig. 1 - Blade without the flexible joint (left). Vertical trajectory $z$ vs. time $t$ (right).

By deriving, first we obtain the velocity $\dot{z}$ and after the acceleration $\ddot{z}$ :

$$
\begin{gathered}
\dot{z}=\frac{z_{\max } \omega}{2} \sin \omega t \\
\ddot{z}=\frac{z_{\max } \omega^{2}}{2} \cos \omega t
\end{gathered}
$$

From the experimental data [1 and 2], it is imposed that the lifting to height $z_{\max }$ equal to $1.3 \mathrm{~m}$ occurs in a time interval $t_{\max }$ of $0.8 \mathrm{~s}$. Therefore the pulsation is $\omega=\pi / t_{\max }=3.925 \mathrm{~s}^{-1}$. Furthermore it is assumed [2] that the average mass is $m$ of $8.2 \mathrm{~kg}$. Finally we neglect the mass of the blade $m_{b}$ and the air drag force $F_{d}$ because of $m_{b} / m \leq 0.05$ and $F_{d} / m g \leq 0.01$.
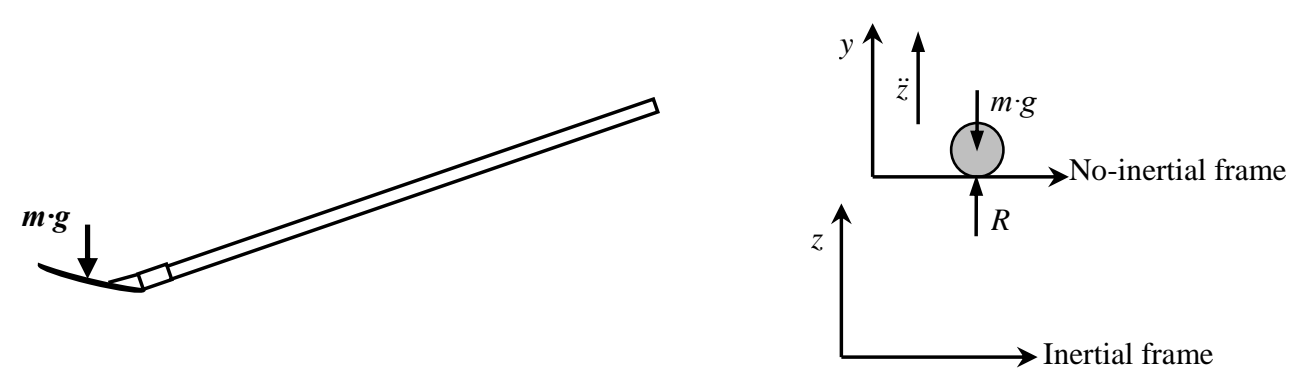

Fig. 2 - Shovel, without the flexible coupling, loaded by $m \cdot g$ (left). Dynamic model (right).

The mass $m$, previously collected from the pile on the ground, is now above the blade (Fig. 2). The blade constitutes an unilateral constraint for the mass $m$. Figure 2 also shows the dynamic model with the inertial frame (ground) [5] and the no-inertial frame identified in the blade subjected to an acceleration $\ddot{z}$ described by eq. (3). The dynamics equation of the mass $m$, considered as a particle, with reference to the no-inertial frame, is: 


$$
m \ddot{y}=-m g+R-m \frac{z_{\max } \omega^{2}}{2} \cos \omega t
$$

Where: $\ddot{y}$ is the acceleration of the mass $m$ with reference to the no-inertial frame (blade); $m g$ is the gravitational force; $R$ is the reaction force; $-m \frac{z_{\max } \omega^{2}}{2} \cos \omega t$ is the fictious force due the accelerated motion of the no-inertial frame.

If the acceleration $\ddot{y}$ is assumed to be zero, through the equation (4) it may obtain the reaction force $R$, which coincides with the force that the blade (and thus the operator) must exert on the mass $m$ to lift it up to $z_{\max }$.
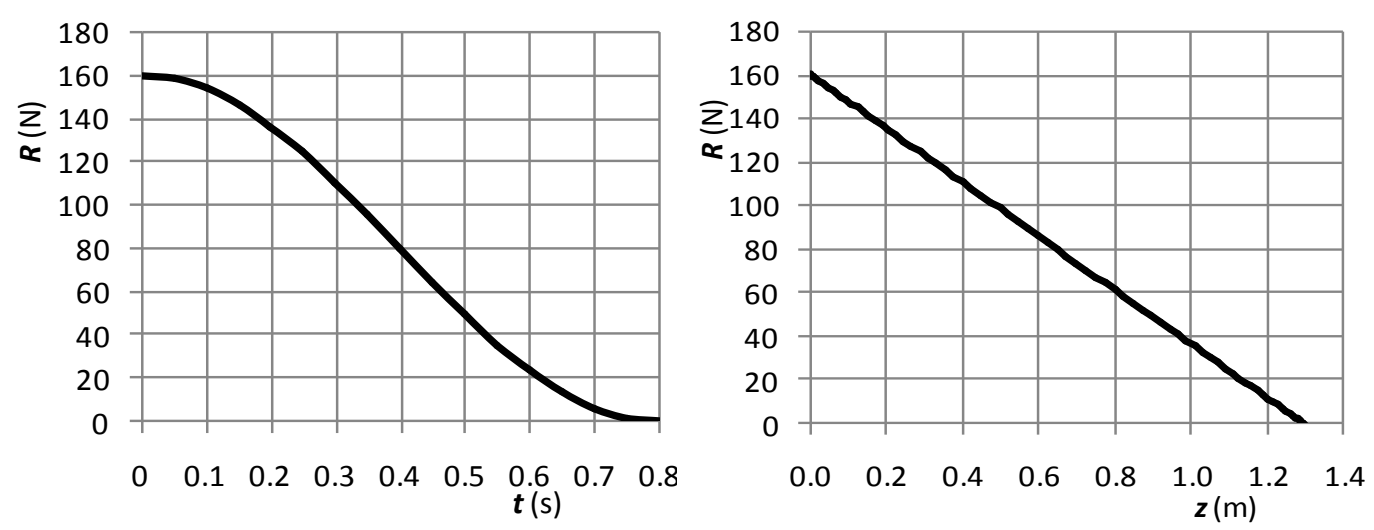

Fig. 3 - Reaction force $R$ coinciding with the force exerted by the operator through the blade on the mass $m$ : vs. time $t$ (left); vs. the lifting $z$ coordinate of both the blade and of the mass $m$ (right).

Figure 3 (left) shows $R=f(t)$ obtained by (4), while figure 3 (right) shows $R=f(z)$ as a result of the combination of (4) with (1). It is easy to see that the area under the graph $R=f(z)$ is the work done by the reaction force on the mass $m$ and therefore is the work done by the operator. Given the linear trend of $R=f(z)$, such work is $W=0.5 \cdot R_{\max } \cdot z_{\max }=0.5 \cdot 160 \cdot 1.3=104 \mathrm{~J}$. It coincides with the potential energy acquired by mass $m$ to reach the maximum height $h_{\max }=z_{\max }=1.3 \mathrm{~m}$ : $W=m g \cdot z_{\max }=80 \cdot 1.3=104 \mathrm{~J}$. This is an obvious result, because it is assumed the presence of only conservative forces. However the diagram in Figure 3 is useful to understand how the force applied by the operator is distributed along the vertical coordinate $z$.

\subsection{Shovel with flexible coupling}

Figure 4 shows the blade with handle with flexible coupling [4]. Both the force of gravity and the force of inertia may cause a deformation of the spring of the coupling. Figure 4 also shows the dynamic model, completed with both inertial frame (ground) and the no-inertial frame, identified in the blade of the shovel imagined without the flexible joint. Therefore the no-inertial frame is still subject to acceleration $\ddot{z}$ as described by eq. (3) and its vertical trajectory $z$ as described 
by eq. (1) and figure 1 . The equation of the dynamics of mass $m$, suspended by the spring of stiffness $k$, with reference to the no-inertial frame, is:

$$
m \ddot{y}=-m g-k y-m \frac{z_{\max } \omega^{2}}{2} \cos \omega t
$$

Where: $\ddot{y}$ is the acceleration of the mass $m$ with reference to the no-inertial frame (blade); $m g$ is the gravitational force; $k y$ is the elastic force; $-m \frac{z_{\max } \omega^{2}}{2} \cos \omega t$ is the fictious force due the accelerated motion of the no-inertial frame; $y$ is the vertical coordinate of the mass $m$ with reference to the no-inertial frame. It is also visible in figure 4 (left) and it is such that the height $h$ of the mass $m$ with reference to the inertial frame is algebraically: $h=z+y$.
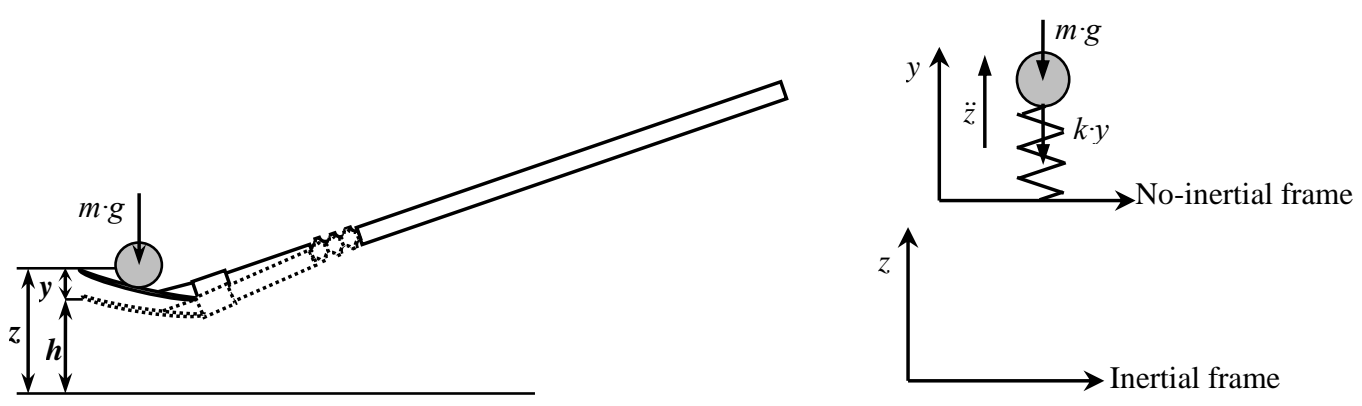

Fig. 4 - Shovel, with the flexible coupling, loaded by $m \cdot g$ (left). Dynamic model (right).

A particular integral of the ODE (5) can be found by placing $y=-\frac{m g}{k}+C \cos \omega t$ that introduced in (5) provides: $C=\frac{0.5 z_{\max }}{1-\frac{\omega_{n}^{2}}{\omega^{2}}}$. Where $\omega_{n}$ is the natural pulsation (frequency) [6, 7, 9 and 10]: $\omega_{n}=\sqrt{\frac{k}{m}}$. Therefore, the particular integral is:

$$
y=-\frac{m g}{k}+\frac{0.5 z_{\max }}{1-\frac{\omega_{n}^{2}}{\omega^{2}}} \cdot \cos \omega t
$$

The general solution of related homogeneous differential equation is:

$$
y=A \cos \omega_{n} t+B \sin \omega_{n} t
$$

Therefore the general solution of the ODE (5) is: 


$$
y=A \cos \omega_{n} t+B \sin \omega_{n} t-\frac{m g}{k}+\frac{0.5 z_{\max }}{1-\frac{\omega_{n}^{2}}{\omega^{2}}} \cos \omega t
$$

To determine the integration constants $A$ and $B$, it must be considered that there is a time range (fig. 5) during which the no-inertial frame lifts, according to the law (1) compressing the spring, but the mass remains fixed $(h=z+y=0)$. The mass will begin lifting when the spring force $k z=-k y$ reaches and exceeds the $m g$ value.
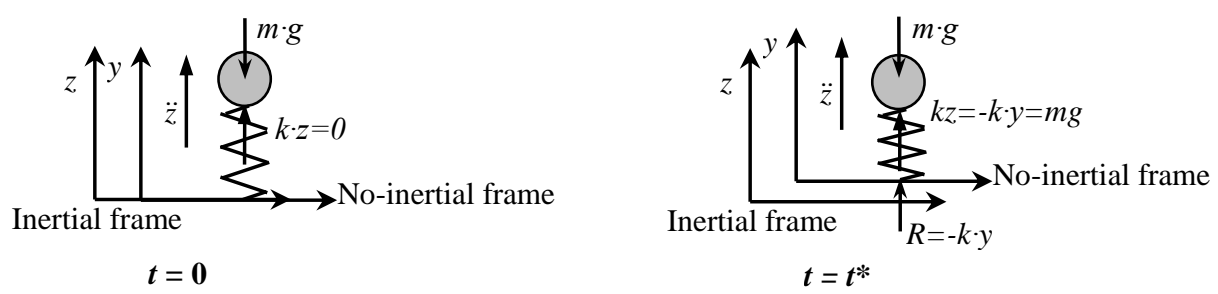

Fig. 5 - Dynamic model during the time range $0-t^{*}$ in which the mass is fixed, but the operator lifts the handle by applying an increasing force $R$ to load the spring.

To find such a time range $0-t^{*}$ we impose, recalling the equation (1), that $m g=0.5 k z_{\max }-0.5 k z_{\max } \cos \omega t^{*}$ :

$$
t^{*}=\frac{1}{\omega} \arccos \left(1-\frac{2 m g}{k z_{\max }}\right)
$$

Therefore the initial conditions of motion $(h=z+y=0)$ and $(\dot{h}=\dot{z}+\dot{y}=0)$, essential to find the value of the constants $A$ and $B$, are applied at time $t^{*}$. A system of two equations in two unknowns $A$ and $B$, is obtained:

$$
\left\{\begin{array}{l}
0=y+z=A \cos \omega_{n} t^{*}+B \sin \omega_{n} t^{*}-\frac{m g}{k}+\frac{0.5 z_{\max }}{1-\frac{\omega_{n}^{2}}{\omega^{2}}} \cos \omega t^{*}+0.5 z_{\max }-0.5 z_{\max } \cos \omega t^{*} \\
0=\dot{y}+\dot{z}=-A \omega_{n} \sin \omega_{n} t^{*}+B \omega_{n} \cos \omega_{n} t^{*}-\frac{0.5 z_{\max } \omega}{1-\frac{\omega_{n}^{2}}{\omega^{2}}} \sin \omega t^{*}+0.5 z_{\max } \omega \sin \omega t^{*}
\end{array}\right.
$$

Solving the system (10):

$$
B=\sin \omega_{n} t^{*}\left[\frac{0.5 z_{\max }}{\frac{\omega^{2}}{\omega_{n}^{2}}-1}\left(\frac{\omega \sin \omega t^{*}}{\omega_{n} \tan \omega_{n} t^{*}}\right)+\frac{m g}{k}-\frac{0.5 z_{\max }}{\frac{\omega^{2}}{\omega_{n}^{2}}-1} \cos \omega t^{*}-0.5 z_{\max }\right]
$$




$$
A=\frac{B}{\tan \omega_{n} t^{*}}-\frac{0.5 z_{\max }}{\frac{\omega^{2}}{\omega_{n}^{2}}-1} \frac{\omega \sin \omega t^{*}}{\omega_{n} \sin \omega_{n} t^{*}}
$$

\section{Results and conclusive discussion}

Considering the case of the mass $m$ is integral (bilateral constraint) with blade of the shovel equipped with a stiffer spring in the flexible coupling, the height $h$ of the mass in motion, obtained by adding $y$ of (8) with $z$ of (1), is shown in figure 6 (left). If the mass $m$ is only put on the blade (unilateral constraint), when $h$ exceeds $z$, for $t=t_{D}$ (in this case $t_{D}$ is $0.407 \mathrm{~s}$ ), then the mass $m$ detaches from the blade.
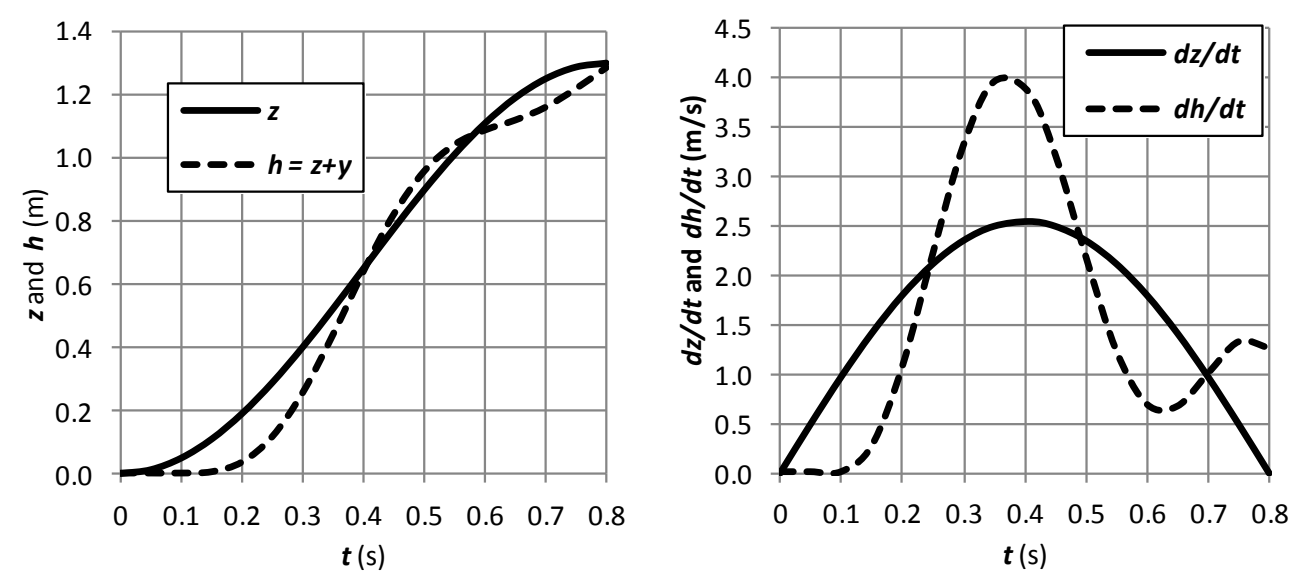

Fig. 6 - Height $h(--)$ of the mass $m$ in motion with bilateral constrain on the shovel with stiffer spring and height $z$ (----) of $m$ on the shovel without springy coupling (left). Their respective velocity $\dot{h}$ and $\dot{z}$ (right).

Figure 6 (right) shows the $m$ mass velocity $\dot{h}=d h / d t=\dot{z}+\dot{y}$, obtained by summing $\dot{y}$, deduced by deriving the equation (8), with the $\dot{z}$ by the equation (2). It provides the speed value $\dot{h}_{D}$ at the time $t_{D}$. Therefore the motion of the mass $m$ after the instant $t_{D}$ results from the integration of the ODE: $m \ddot{h}=-m g$, with initial condition: $\dot{h}=\dot{h}_{D}$ and $h=h_{D}$ for $t=t_{D}$ :

$$
h=h_{D}+\dot{h}_{D}\left(t-t_{D}\right)-0.5 g\left(t-t_{D}\right)^{2} \quad \dot{h}=\dot{h}_{D}+g\left(t-t_{D}\right)
$$

Therefore in the time range $0-t_{D}$, the $h$ values of the mass $m$ unilaterally constrained, are obtained by $h=z+y$ (fig. 6), while, after $t_{D}$, the value of $h$ is provided by equation (13). Figure 7 (left) shows the $h$ values vs. time, while figure 7 (right) shows the corresponding speed. 

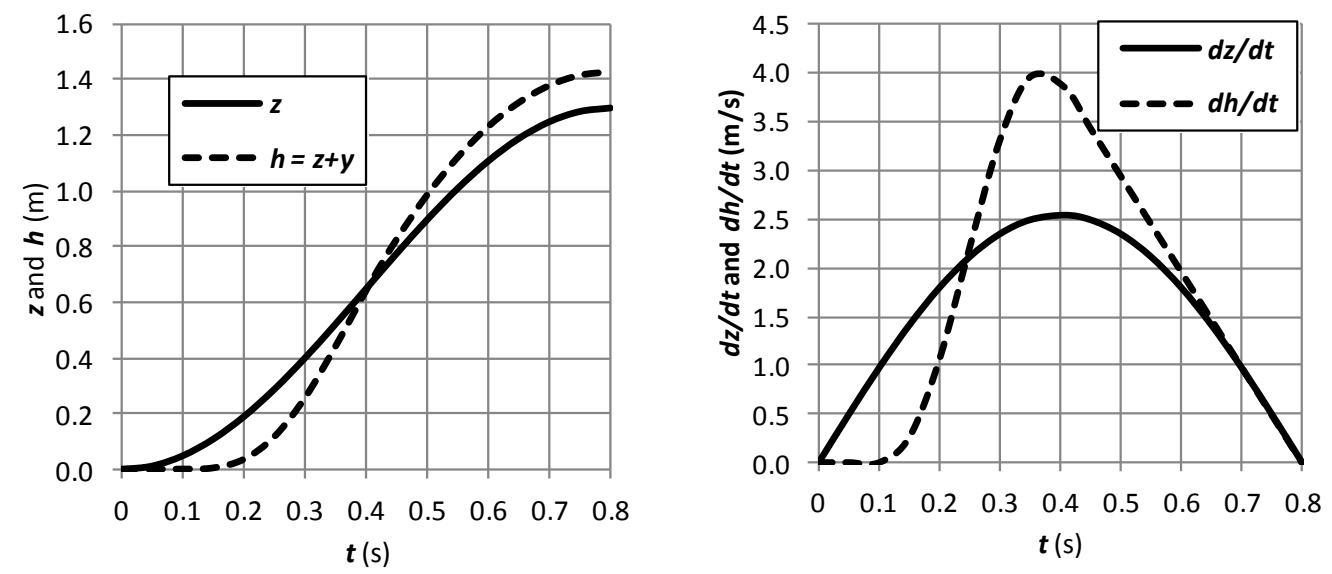

Fig. 7 - Height $h(--)$ of the mass $m$ in motion with unilateral constrain on the shovel with stiffer spring and height $z$ (----) of $m$ on the shovel without springy coupling (left). The corresponding velocities $\dot{h}$ and $\dot{z}$ (right).
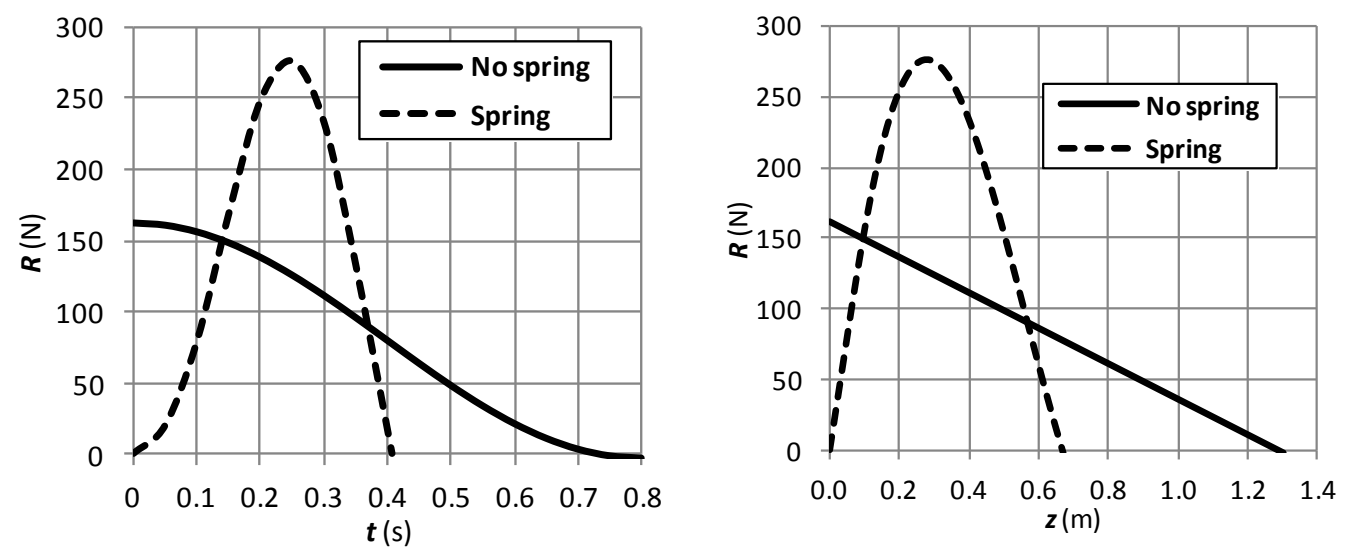

Fig. 8 - Force $R(t)$ (- - ) exerted by the operator through the shovel with stiffer spring vs. the $R(t)$ (----) exerted through the shovel without flexible coupling (left); Force $R(z)$ (- - -) exerted by the operator through the shovel with stiffer spring vs. the $R(z)(---)$ exerted through the shovel without flexible coupling (right).

For reasons of balance, figure 5 (right) shows that the blade (no-inertial frame) handled by the operator, exerts a reaction force $R$ coinciding with the elastic force $-k y$. Therefore $R$ coincides with the force that the operator must exert and it can be calculated by multiplying the stiffness $k$ for the equation (8), obtaining $R=f(t)$ (fig. 8 -left). Combining (8) with (1) we get the reaction force $R$ (Fig. 8-right) vs. the height $z$ of handling of the operator, coinciding with the height of the non-inertial frame.

As already seen with figure 3, also in this case the area under the diagram $R=f(z)$ is the work done by the reaction force $R$ on the mass $m$ and therefore is the work done by the operator. Also in this case, due to the presence of only conservative 
forces, it coincides with the potential energy acquired by the mass $m$ to the achievement of the maximum height $h_{\max }: W=m g \cdot h_{\max }=80 \cdot 1.424=114 \mathrm{~J}$.

The diagrams in figure 8 allow to understand that the force $R$, in presence of the flexible coupling (stiffer spring), is exerted by the operator only in the first section of the lifting, but with a peak value $(275 \mathrm{~N})$ greater than that of the shovel without flexible coupling $(160 \mathrm{~N})$. This is because of the need to load the spring. However, two advantages are obtained: first, a $10 \%$ increase of the height reached by the mass $m$; second, the reaction force $R$ is exerted within the first $0.64 \mathrm{~m}$ from the ground (about $z_{\max } / 2$ ) when the operator can leverage the thigh as a support for the handle of the shovel, with a presumable less load on the spine than the shovel without spring.

In case of a shovel with semi-stiff spring, an increase of total height $h_{\max }$ reached by the mass $m$ of $29 \%(1.675$ vs. $1.3 \mathrm{~m})$ and a peak value of the reaction force $R$ slightly lower (268 vs. $275 \mathrm{~N}$ ) are obtained. Conversely the operator effort, represented by $R$, will be exerted within the first $0.8 \mathrm{~m}$ from the ground, instead of $0.64 \mathrm{~m}$, but always lower than $1.3 \mathrm{~m}$ of the blade without spring.

It is interesting to compare the trajectories of the mass $m$, if the operator produces a horizontal initial speed of $2 \mathrm{~m} / \mathrm{s}$ of $m$ at the time when $m$ leaves the blade, by manoeuvring the handle. Figure 9 shows how the horizontal distance that the mass $m$ can cover before falling to a final height of $1 \mathrm{~m}$ (hypothetical height of the flatcar). It grows from 0.49 , for the shovel without spring, at $1.37 \mathrm{~m}$ for the shovel with stiffer spring, with an increase of $180 \%$.

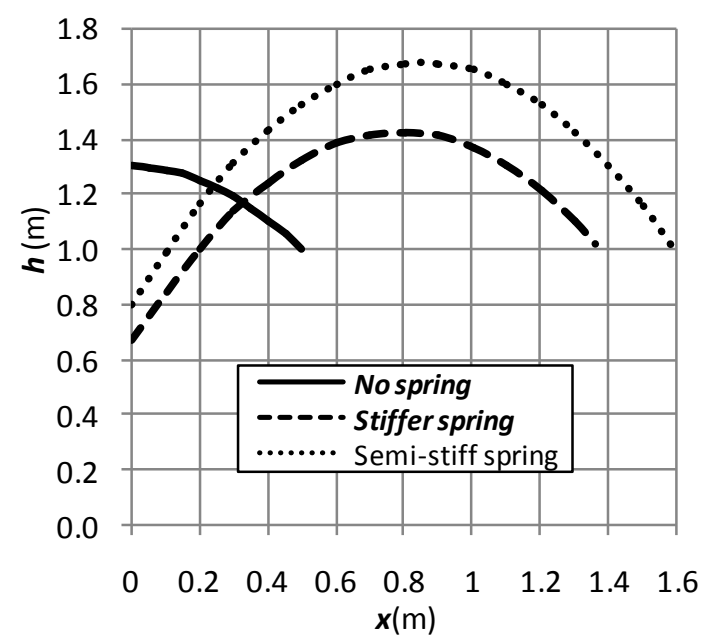

Fig. 9 - Trajectories of the mass $m$ under the effect of a horizontal initial speed $\dot{x}_{0}=2 \mathrm{~m} / \mathrm{s}$, in the three cases of shovel: without spring; with stiffer spring; with semi-stiff spring.

\section{References}

[1] R.S. Bridger, N. Cabion, J. Goedecke, S. Rickard, E. Schabort, C. Westgarth-Taylor, M.I. Lambert, Physiological and subjective measures of 
workload when shovelling with a conventional and two-handled shovel, Ergonomics, 40 (1997), no. 11, 1212 - 1219.

http://dx.doi.org/10.1080/001401397187450

[2] U. Glitsch, H.J. Ottersbach, R. Ellegast, K. Sawatzki, J. Voss, A. Luttmann M. Jager, G. Reheme, Belastung der Lendenwirbelsäule bei Schaufeltätigkeiten, BGIA - Report 4-2008.

[3] Patent n. EP 2308653 (A2) - Shovel (13-04-2011) and EP 2308653 (A3) Shovel (13-01-2013). Inventor: Haller Hans-Peter.

[4] D. Friso, M. Bietresato, Dynamic Analysis and Design Guidelines of Mechanical Oscillators for Cutting Soil Through Vibrating Tools, Journal of Vibroengineering, 14 (2012), no. 4, 1775 - 1786.

[5] P. Sinha, A Re-Look at the Classical Laws of Motion, Advances in Applied Physics, 6 (2012), no. 20, 969 - 974.

[6] A. Kargar, M. Akbarzade, Frequency Analysis of Large-Amplitude Oscillation of a Rotational Pendulum System Using He's Amplitude-Frequency Formulation (HFAF) and He's Energy Balance Method (HEBM), International Journal of Mathematical Analysis, 6 (2012), no. 23, 1147 1152.

[7] N. T. Alshabatat, Beam Natural Frequency Tuning Using V-Notches, Contemporary Engineering Sciences, 5 (2012), no. 2, 49 - 57.

[8] D. Friso, A New Mathematical Model for Food Thermal Process Prediction Modelling and Simulation in Engineering, 2013 (2013), 1 - 8. N. 569473 http://dx.doi.org/10.1155/2013/569473

[9] A. Khanna, N. Kaur, Effect of Thermal Gradient on Natural Frequencies of Tapered Rectangular Plate, International Journal of Mathematical Analysis, 7 (2013), no. 16, 755 - 761.

[10] A. Zaryankin, N. Rogalev, A. Rogalev, A. Kocherova, W. Strielkowski, Line Summary of Approaches for Improving Vibrational Reliability of Thermomechanical Equipment and Its Interconnecting Pipelines at Thermal Power Plant, Contemporary Engineering Sciences, 7 (2014), no. 33, 1793 1806. http://dx.doi.org/10.12988/ces.2014.410190

[11]L.V. Makarova, R.V.Tarasov, D.V. Tarasov, A Methodical Approach to Ensure the Stability and Quality of Technological Processes, Contemporary Engineering Sciences, 8 (2015), no. 6, 257 - 261.

http://dx.doi.org/10.12988/ces.2015.5125 
[12]D. Friso, C. Baldoin, F. Pezzi, Mathematical Modeling of the Dynamics of Air Jet Crossing the Canopy of Tree Crops during Pesticide Application Applied Mathematical Sciences, 9 (2015), no. 26, 1281 - 1296. http://dx.doi.org/ 10.12988/ams.2015.5145

[13]D. Friso, A Mathematical Solution for Food Thermal Process Design, Applied Mathematical Sciences, 9 (2015), no. 6, 255 - 270. http://dx.doi.org/10.12988/ams.2015.411980

Received: June 29, 2015; Published: August 25, 2015 